\title{
New Insights Into the Effects of Individual Chinese Herbal Medicines on Chronic Kidney Disease
}

\author{
Minghai Shao ${ }^{1}$, Chaoyang $\mathrm{Ye}^{1}$, George Bayliss ${ }^{2}$ and Shougang Zhuang $^{2,3 *}$ \\ ${ }^{1}$ Department of Nephrology, Shuguang Hospital, Shanghai University of Traditional Chinese Medicine, Shanghai, China, \\ ${ }^{2}$ Department of Medicine, Rhode Island Hospital and Alpert Medical School, Brown University, Providence, RI, United States, \\ ${ }^{3}$ Department of Nephrology, Shanghai East Hospital, Tongji University School of Medicine, Shanghai, China
}

OPEN ACCESS

Edited by: Dan-Qian Chen,

Northwest University, China

Reviewed by:

Yifei Zhong,

Longhua Hospital, China

Yue Tu,

Nanjing University of Chinese Medicine, China

*Correspondence:

Shougang Zhuang

szhuang@lifespan.org

Specialty section:

This article was submitted to

Renal Pharmacology,

a section of the journal

Frontiers in Pharmacology

Received: 11 September 2021

Accepted: 20 October 2021

Published: 04 November 2021

Citation:

Shao M, Ye C, Bayliss G and Zhuang S (2021) New Insights Into the Effects of Individual Chinese Herbal Medicines

on Chronic Kidney Disease.

Front. Pharmacol. 12:774414.

doi: 10.3389/fphar.2021.774414
The clinical and experimental study into the effects of Chinese herbal medicines on chronic kidney disease has evolved over the past 40 years with new insight into their mechanism and evidence of their clinical effects. Among the many traditional Chinese herbs examined in chronic renal disease, five were found to have evidence of sufficient clinical efficacy, high frequency of use, and well-studied mechanism. They are: Abelmoschus manihot and Huangkui capsule, Salvia miltiorrhiza and its components (tanshinone II A, salvianolic acid A and B); Rhizoma coptidis and its monomer berberine; Tripterygium wilfordii and its components (triptolide, tripterygium glycosides); Kudzu root Pueraria and its monomer Puerarin. These Chinese herbal medications have pharmaceutical effects against fibrosis, inflammation and oxidative stress and also promote renal repair and regeneration. This article reviews their clinical efficacy, anti-fibrotic effects in animal models, and molecular mechanism of action.

Keywords: renal fibrosis, Chinese herbs, monomers, chronic kidney disease, Abelmoschus manihot, Salvia miltiorrhiza, therapeutic molecular mechanism

\section{INTRODUCTION}

Chronic kidney disease (CKD) is a global public health issue, affecting more than $10 \%$ of the world's population (Glassock et al., 2017; Ruiz-Ortega et al., 2020). The burden of CKD is not only restricted to the requirement of renal replacement therapy for end stage of renal disease (ESRD), but also associated with cardiovascular events and mortality (Glassock et al., 2017). Although the etiology of $\mathrm{CKD}$ and pathological course are diverse, renal interstitial fibrosis and gradual loss of nephron mass are the common pathological changes. Renal fibrosis is characterized by activation of renal interstitial fibroblasts and deposition of extracellular matrix components that are driven by multiple signaling pathways, transcriptional factors, inflammatory factors, oxidative stress and vasoactive substances, including angiotensin (Liu 2011; Meng, et al., 2016; Ruiz-Ortega, et al., 2020). Current treatment of patients with CKD most still relies mostly on an angiotensin-converting enzyme inhibitor (ACEI) and an angiotensin receptor blocker (ARB), however, these drugs only ameliorate, but not halt the progression of CKD to ESRD (Ruiz-Ortega et al., 2020). The limitations of Western medicine in curing or slowing progression of CKD may drive some patients to seek alternative treatments such as Chinese herbal medicines.

Chinese herbal medicines have been extensively used to treat CKD and other chronic diseases in China and some Asian countries. However, high-quality clinical evidence is lacking to support use of Chinese herbal medicines for CKD treatment worldwide. In 2015, Lin et al., published the first population-based retrospective cohort study on the use of Chinese herbal medicine in CKD patients 
(Lin et al., 2015). By searching for the Taiwan National Health Insurance Research Database from 2000 to 2005, they found that among the 24,971 study patients, 11,351 received prescribed Chinese herbal medicine after CKD diagnosis. After adjusting confounding variable, the group using Chinese herbal medicine exhibited a significantly reduced ESRD risk (60\%) compared with the nonuse group. This provides solid evidence of the association between the use of Chinese herbal medicines with reduced ESRD risk in patients with CKD. Further analysis of Chinese herbal medicines used in this population of CKD patients revealed that the formulas classified as "blood-regulating," "dampnessdispelling," or "harmonizing" were strongly associated with the protection effect against CKD (Lin et al., 2015), suggesting that these classes of Chinese herbal formulas contain therapeutic components that prevent CKD progression.

A Chinese herbal medicine formula usually contains several medicinal herbs. Identifying the role of individual herbs is essential for understanding the role and mechanism of a Chinese herbal medicine formula in treating various diseases including CKD. In the past 40 years, many such studies have been conducted to search for medicinal herbs that are effective for treating CKD. Five were found to have evidence of sufficient clinical efficacy, high frequency of use, and well-studied mechanism. These herbs include Abelmoschus Manihot, Salvia miltiorrhiza, Rhizoma coptidis, Tripterygium wilfordii, and Kudzu root Pueraria. Interestingly, these five Chinese medicinal herbs are also major components in either blood-regulating, dampnessdispelling, heat-clearing, or harmonizing formula associated with beneficial effect to CKD patients as mentioned above (Lin et al., 2015). Moreover, the extract and/or monomer of these five medicinal herbs have been made and tested in animal models of CKD and/or patents with CKD (see below). A prospective, open-label, multicenter, randomized controlled trial demonstrated that Huangkui capsule, a single-plant drug extracted from the dry corolla of Flos A. Manihot, was more effective than the angiotensin-receptor blocker losartan in reducing proteinuria in patients with primary glomerular disease after 24 weeks of treatment (Zhang, et al., 2014), which resulted in its approval by the China Food and Drug Administration to treat CKD stages 1-2 with primary glomerular disease.

In this article, we review the therapeutic effect of these five medicinal herbs in animal models of CKD (Table 1) and their clinical efficacy in CKD patients (Table 2) as well as molecular mechanism of their actions. We also discuss the challenge and directions of medicinal herb research associated with CKD.

\section{ABELMOSCHUS MANIHOT AND HUANGKUI CAPSULE}

Abelmoschus manihot, also called as "Huangkui" in Chinese, is an annual flowering herb plant in the family of Malvaceae. As a traditional Chinese medicine (TCM), the ethanol extract of the flower in Abelmoschus manihot is made as Huangkui capsule and has been used for medication of the patients with kidney diseases. Studies have confirmed that the major pharmacologically bioactive constituents in the flower of Abelmoschus Manihot are seven flavonoids, including Rutin, Hyperoside, Hibifolin, Isoquercetin, Myricetin, Quercetin, and Quercetin-3-Orobinobioside (Guo, et al., 2015a).

\section{Animal Studies}

The biological effects of Abelmoschus manihot have been studied in several animal models of CKD, including 5/6 nephrectomy (Gu, et al., 2020), adriamycin-induced nephropathy (Li, et al., 2019), and streptozotocin-induced diabetic nephropathy (DN) (Liao, et al., 2019). The overall results show that treatment with Abelmoschus Manihot can improve kidney function, attenuate kidney damage and tubulointerstitial fibrosis, and reduce proteinuria (Cai, et al., 2017). These beneficial effects are related to inhibition of inflammation (Li, et al., 2019), antioxidative stress (Liao, et al., 2019), inhibiting renal epithelialmesenchymal transition (EMT) (Gu, et al., 2020; Peng, et al., 2016), remodeling the intestinal microbiota and inhibiting microinflammation (Tu, et al., 2020). Mechanistically, Abelmoschus Manihot is able to suppress ROS-ERK1/2-mediated NLRP3 (NLR Family Pyrin Domain Containing 3) inflammasome activation (Li, et al., 2019), reduce tumor necrosis factor- $\alpha$ (TNF- $\alpha$ ) and transforming growth factor- $\beta 1$ (TGF- $\beta 1$ ) protein expression ( $\mathrm{Tu}$, et al., 2013), inhibiting p38MAPK signaling pathway (Tu, et al., 2013) and autophagy-mediated macrophage polarization ( $\mathrm{Tu}$, et al., 2020). Abelmoschus manihot can also prevent glomerular podocyte apoptosis (Zhou, et al., 2012) by a mechanism associated with activating peroxisome proliferator-activated receptor (PPAR)-alpha/gamma (Ge, et al., 2016), inhibiting iRhom2/TACE signaling pathway (Liu, et al., 2017), attenuating endoplasmic reticulum stress (ERS) (Ge, et al., 2016; Liu, et al., 2017), and regulating autophagy and mitochondrial dynamics (Kim, et al., 2018). Moreover, Abelmoschus manihot has an ability to reduce oxidative stress and inflammation via modulation of AMPK (AMP-activated protein kinase)-Sirt1-PGC-1a (peroxisome proliferatoractivated receptor-gamma coactivator- $\alpha$ ) signaling axis (Liao, et al., 2019) and NADPH oxidase/ROS/ERK pathway (Cai, et al., 2017).

\section{Clinical Studies}

Abelmoschus manihot is one of the important drugs for the treatment of CKD. It has been reported that treatment with Abelmoschus manihot can reduce proteinuria and improve renal function in patients with diabetic kidney disease (DKD) (Shi, et al., 2019), IgA nephropathy (Li, et al., 2020a), and CKD stages 1-2 (Zhang, et al., 2014). In a meta-analysis identified 72 clinical investigations involving 5,895 participants. Compared to a RAS blocker alone, combined treatment of Abelmoschus Manihot with a RAS blocker was more effective in reducing $24 \mathrm{~h}$ urinary protein $(24 \mathrm{~h} \mathrm{UP})$, urinary albumin excretion rate (UAER), and serum creatinine (SCr) levels. Abelmoschus manihot did not increase adverse events (Shi, et al., 2019). Recently, a multicenter randomized controlled clinical trial for determining the efficacy of Abelmoschus Manihot were conducted in a total of 417 patients with biopsy-proven primary glomerular disease (Zhang, et al., 2014) (CKD stage 
TABLE 1 | Recent animal studies on Chinese herbal medicines with anti-renal fibrosis function.

\begin{tabular}{|c|c|c|c|c|}
\hline Herbal/extract & Animal model & Outcome & Mechanism & Reference \\
\hline Abelmoschus & 5/6 nephrectomy & $\downarrow$ EMT & $\downarrow$ PI3K-Akt-eNOS, ERK1/2 & Gu, et al. (2020); Peng, et al. (2016) \\
\hline \multirow[t]{6}{*}{ Manihot } & ADRN & $\downarrow O X$, inflammation & $\begin{array}{l}\downarrow \text { ROS-ERK } 1 / 2-N L R P 3 \\
\downarrow N A D P H \text { oxidase/ROS/ERK }\end{array}$ & Li, et al. (2019); Cai, et al. (2017) \\
\hline & UNE-ADR & \inflammation, glomerulosclerosis & $\downarrow$ TNF- $\alpha$, TGF- $\beta 1$, p38MAPK & Tu, et al. (2013) \\
\hline & STZ-DN mice & $\downarrow O X$ & $\uparrow A M P K-S i r t 1-P G C-1$ & Liao, et al. (2019) \\
\hline & UNE-STZ-DN & $\downarrow$ podocyte apoptosis & $\begin{array}{l}\uparrow P P A R-\alpha / \gamma \\
\downarrow \text { iRhom } 2 / T A C E, E R S\end{array}$ & Ge, et al. (2016); Liu, et al. (2017) \\
\hline & $\begin{array}{l}\text { UNE-STZ- } \\
\text { HFD-DN }\end{array}$ & $\downarrow$ podocyte loss, FN & $\begin{array}{l}\text { Regulating autophagy, } \\
\text { mitochondrial dynamics }\end{array}$ & Kim, et al. (2018) \\
\hline & UPPR rat & $\begin{array}{l}\text { †intestinal microbiota } \\
\downarrow \text { micro-inflammation }\end{array}$ & $\begin{array}{l}\text { autophagy-mediated macrophage } \\
\text { polarization }\end{array}$ & Tu, et al. (2020) \\
\hline $\begin{array}{l}\text { Salvianolic Acid A, } \\
\text { Tanshinone IIA }\end{array}$ & 5/6 nephrectomy & $\downarrow O X$, inflammation & $\begin{array}{l}\uparrow A k t / G S K-3 \beta / N r f 2, \text { BMP-7, Smad6 } \\
\downarrow N F-k B, \text { p38 MAPK, TGF- } \beta / \text { Smads }\end{array}$ & $\begin{array}{l}\text { Zhang, et al. (2019b); Zhang, et al. } \\
\text { (2019a); Zhang, et al. (2018); Wang, } \\
\text { et al. (2015b) }\end{array}$ \\
\hline Salvianolic acid A & ADR-MCD rats & $\downarrow$ proteinuria, podocyte injury & $\uparrow P P A R-\gamma /$ Angptl4, Nrf2/HO-1 & Wang, et al. (2019b) \\
\hline Tanshinone IIA & STZ-DN & $\begin{array}{l}\downarrow E R S, \text { albuminuria } \\
\downarrow \text { pathological damage }\end{array}$ & $\begin{array}{l}\downarrow \text { PERK } \\
\downarrow \mathrm{OX}, \text { inflammation }\end{array}$ & $\begin{array}{l}\text { (Xu, et al., 2020a) } \\
\text { Chen, et al. (2017) }\end{array}$ \\
\hline Salvianolic Acid A & STZ-HFD-DN & $\begin{array}{l}\downarrow \text { OX, inflammation, endothelial permeability; } \\
\text { †autophagy }\end{array}$ & $\begin{array}{l}\downarrow \text { AGE-RAGE-RhoA/ROCK, AGE- } \\
\text { RAGE-Nox4 axis }\end{array}$ & Hou, et al. (2017) \\
\hline Salvianolic Acid B & UUO rat & $\downarrow$ pathological damage & \heparanase/syndecan 1 & Hu, et al. (2020) \\
\hline Salvianolic Acid A, C & UUO rat & $\begin{array}{l}\text { Trenal function, tubular function } \\
\downarrow \text { pathological damage }\end{array}$ & $\downarrow$ CCL5 and CXCL10 & Li, et al. (2015) \\
\hline Tanshinone ॥A & AD-PO-UAN & $\downarrow 0 x$ & $\downarrow$ NOX4, MAPK & Zhang et al. (2020c) \\
\hline Salvianolic Acid B & $\mathrm{RIRI}$ rats & $\begin{array}{l}\downarrow \mathrm{OX} \text {, inflammation; caspase-1-mediated } \\
\text { pyroptosis }\end{array}$ & $\uparrow \mathrm{PI3K} /$ Akt; $\downarrow$ Nrf2 pathway & $\begin{array}{l}\text { Ma et al., (2017b) } \\
\text { Pang, et al. (2020) }\end{array}$ \\
\hline Tanshinone IIA & ioversol-CIN & $\downarrow$ tubular necrosis, apoptosis, OX & $\uparrow$ Nrf2/ARE activation & Liang, et al. (2018) \\
\hline Tanshinone I & $\mathrm{AAl}-\mathrm{KI}$ & $\downarrow k i d n e y$ injury & $\uparrow$ cytochrome P450 1A & Feng, et al. (2013) \\
\hline Salvianolic Acid B & FA-RTI mice & $\downarrow$ tubular injury & $\downarrow$ ERS & Mai, et al. (2020) \\
\hline \multirow[t]{9}{*}{ Berberine } & UUO rats & \ECM, inflammation, OX & $\downarrow$ TGF-b1/Smad3 & Wang, et al. (2014) \\
\hline & DKD Murine & $\begin{array}{l}\text { improve metabolism; \podocyte damage, } \\
\text { glomerulosclerosis, mitochondrial dysfunction }\end{array}$ & $\begin{array}{l}\downarrow \text { mitochondrial ROS } \\
\uparrow P G C-1 a\end{array}$ & Qin, et al. (2020) \\
\hline & STZ-DN & $\downarrow$ proteinuria, TIF, podocytes injury & 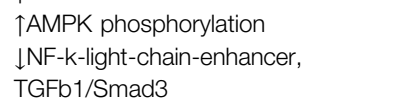 & $\begin{array}{l}\text { Sun et al. (2015); Zhang et al. (2020b) } \\
\text { Li and Zhang, (2017) }\end{array}$ \\
\hline & & & $\uparrow D r p 1$ & Qin, et al. (2019) \\
\hline & STZ-DN & \inflammation & $\downarrow T L R 4 / N F-k B$ & $\begin{array}{l}\text { Sun et al. (2015) } \\
\text { Zhu et al. (2018) }\end{array}$ \\
\hline & STZ-DN & $\downarrow \mathrm{ECM}$ & $\downarrow N r f 2 ;$ regulating MMPs/TIMPs & $\mathrm{Ni}$, et al. (2015) \\
\hline & STZ-DN & †renal pathology & $\uparrow G R K s$ & Wang, et al. (2013) \\
\hline & SHR & \hypertension, renal damage & 」RAS, IL-6, IL-17, IL-23 & Guo, et al. (2015b) \\
\hline & $\begin{array}{l}2 \mathrm{~K} 1 \mathrm{C}-\mathrm{RV}-\mathrm{HTN} \\
\text { rats }\end{array}$ & $\downarrow$ hypertension, sympathoexcitation & ROS/Erk1/2/iNOS & Tian, et al. (2019) \\
\hline $\begin{array}{l}\text { tripterygium } \\
\text { glycosides }\end{array}$ & NUE-STZ-DN & $\downarrow$ glomerulosclerosis, TIF, microinflammation & $\begin{array}{l}\text { \macrophage infiltration, TNF-a, IL- } \\
\text { 1b, TGF-b1, p38 MAPK, NF-kB }\end{array}$ & Wu, et al. (2017) \\
\hline \multirow[t]{8}{*}{ Triptolide } & STZ-HFD-DN & $\downarrow M A$, inflammation, pathological damage & $\begin{array}{l}\text { regulating Th cell balance } \\
\downarrow \text { macrophage infiltration }\end{array}$ & Guo et al. (2016) \\
\hline & STZ-HFD-DN & \renal EMT & MiR-188-5p-PI3K/AKT & Xue et al. (2018) \\
\hline & STZ-DN & \renal ECM & $\downarrow$ microRNA-137/Notch1 & Han, et al. (2018) \\
\hline & STZ-DN & restoring autophagy & $\downarrow m i R-141-3 p / P T E N / A k t / m T O R$ & Li, et al. (2017b) \\
\hline & UUO rats & \inflammatory, ECM; immune activity & $\begin{array}{l}\downarrow \text { TGF- } \beta 1, \text { CTGF, MCP1, } \\
\text { osteopontin }\end{array}$ & Yuan, et al. (2011) \\
\hline & PKD adult rats & $\downarrow$ disease progression; †renal function & \JAK2-STAT3 & Jing, et al. (2018) \\
\hline & $\begin{array}{l}\text { DOCA-salt } \\
\text { hypertension }\end{array}$ & $\downarrow$ pathological damage & \inflammatory & Zhang, et al. (2020a) \\
\hline & FSGS rats & $\downarrow$ kidney injury, podocyte apoptosis & $\downarrow \| \mathrm{L} 4$ & Li, et al. (2020c) \\
\hline \multirow[t]{5}{*}{ Puerarin } & UUO murine & $\downarrow E C M$, TIF, epithelial cell apoptosis & $\begin{array}{l}\downarrow N O X 4 ; \text { p phosphorylation of p38, } \\
\text { ERK, JNK, MAPK }\end{array}$ & Zhou, et al. (2017) \\
\hline & $\begin{array}{l}\text { STZ-DM } \\
\text { eNOS(-/-) mice }\end{array}$ & $\downarrow O X$, albuminuria, kidney injury & 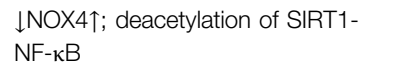 & Li et al. (2017a); Xu, et al. (2020c) \\
\hline & STZ-DN & \pathological damage, apoptosis & 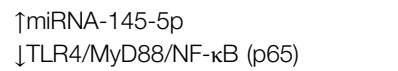 & Li et al. (2017a); Xu, et al. (2020c) \\
\hline & STZ- DN mice & $\downarrow U A C R$, kidney injury & $\begin{array}{l}\uparrow H M O X 1, \text { Sirt1-mediated podocyte } \\
\text { autophagy }\end{array}$ & Li, et al. (2020b) \\
\hline & STZ- DN rats & $\downarrow$ kidney hypertrophy, OX, podocyte injury & $\begin{array}{l}\text { †nephrin, podocin } \\
\downarrow \text { MMP9 }\end{array}$ & Zhong, et al. (2014) \\
\hline
\end{tabular}


TABLE 1 | (Continued) Recent animal studies on Chinese herbal medicines with anti-renal fibrosis function.

\begin{tabular}{|c|c|c|c|c|}
\hline Herbal/extract & Animal model & Outcome & Mechanism & Reference \\
\hline & STZ-DN mice & †autophagy, nephrin, podocin, podocalyxin & $\begin{array}{l}\uparrow \text { PERK/elF2 } \alpha / \text { ATF4 } \\
\downarrow H I F-1 a l p h a, \text { VEGF }\end{array}$ & $\begin{array}{l}\text { Xu et al. (2020b) } \\
\text { Shukla, et al. (2017) }\end{array}$ \\
\hline & STZ-DN rats & $\downarrow$ renal AGEs contents & $\downarrow$ AGEs, RAGE & Shen, et al. (2009) \\
\hline
\end{tabular}

$\uparrow$ : increase or activation or improve; $\downarrow$ : decrease or inhibition; AAl: aristolochic acid l; ADR: Adriamycin; ADRN: adriamycin nephropathy; AD-PO-UAN: adenine and potassium oxonateinduced uric acid nephropathy mice; AMPK: AMP-activated protein kinase; BMP-7: bone morphogenetic protein 7; CTGF: connective tissue growth factor; CIN: Contrast-Induced Nephropathy; DN: diabetic nephropathy; DM: diabetes mellitus; DKD: diabetic kidney disease; Drp1: dynamin-related protein 1; ECM: extracellular matrix; EMT: epithelial-mesenchymal transition; EMTD: epithelial-myofibroblast trans-differentiation; ERS: endoplasmic reticulum stress; eNOS(-/-): endothelial nitric oxide synthase-null mice; FA-RTI: fatty acids-induced renal tubular injury; FSGS: focal segmental glomerular sclerosis; GRKs: G protein-coupled receptor kinases; HFD: high-fat diet; KI: kidney injury; MMPs: matrix metalloproteinases; MCD: minimal change disease; NF: nuclear factor; NLRP3: NLR Family Pyrin Domain Containing 3; OX: oxidative stress; PGC-1: peroxisome proliferator-activated receptor-gamma coactivator-1; PKD: polycystic kidney disease; PO: potassium oxonate; PPAR: peroxisome proliferator-activated receptor; RIRI: renal ischemia-reperfusion injury; SHR: spontaneously hypertensive rats; STZ: Streptozotocin; STZ-DN: streptozotocin induced diabetic nephropathy; STZ-HFD-DN: streptozotocin induced and high-fat diet diabetic nephropathy; UACR: urinary albumin creatinine ratio; MA: urine micro-albumin; UNE: unilateral nephrectomy; UPPR: rat models were induced by uninephrectomy, potassium oxonate, and proinflammatory diet; UUO: unilateral ureteral obstruction; TIF: tubulointerstitial fibrosis; TIMPs: tissue inhibitor of metalloproteinases; TNF-a: tumor necrosis factor-a; TGF-b1: transforming growth factorb1; 2K1C-RV-HTN:Two-kidney, one-clip renovascular hypertensive rats.

\begin{tabular}{|c|c|c|c|c|c|c|}
\hline $\begin{array}{l}\text { Chinese herbal } \\
\text { name }\end{array}$ & Disease & $\mathbf{N}$ & Therapeutic arms & Primary outcome & $\begin{array}{l}\text { Study } \\
\text { period }\end{array}$ & References \\
\hline \multirow[t]{3}{*}{$\begin{array}{l}\text { Abelmoschus Manihot } \\
\text { (Huangkui capsule) }\end{array}$} & $\begin{array}{l}\operatorname{IgAN}(24 \mathrm{hUTP} 0.5-3.0 \mathrm{~g} / \mathrm{d} \\
\left.\text { eGFR } \geq 45 \mathrm{ml} / \mathrm{min} / 1.73 \mathrm{~m}^{2}\right)\end{array}$ & 1,470 & $\begin{array}{l}\text { Huangkui capsule/placebo vs. losartan } \\
\text { placebo }\end{array}$ & 24 h UTP (mg/d) & 48 weeks & Li, et al. (2020a) \\
\hline & DN & 5,895 & $\begin{array}{l}\text { Huangkui capsule + RAS blocker vs. RAS } \\
\text { blocker }\end{array}$ & $\begin{array}{l}24 \text { hUTP }(\mathrm{g} / \mathrm{d}) ; \text { UAER } \\
\text { (mug/min); } \\
\text { SCr(umol/L) }\end{array}$ & $\begin{array}{l}\text { Meta- } \\
\text { Analysis }\end{array}$ & Shi, et al. (2019) \\
\hline & $\begin{array}{l}\text { CKD1-2, primary glomerular } \\
\text { disease (biopsy), moderate } \\
\text { proteinuria }\end{array}$ & 417 & $\begin{array}{l}\text { Huangkui capsule vs. losartan vs. Huangkui } \\
\text { capsule + losartan }\end{array}$ & 24 hUTP (mg/d) & 24 weeks & $\begin{array}{l}\text { Zhang, et al. } \\
\text { (2014) }\end{array}$ \\
\hline Tanshinone & CKD & 1,857 & Tanshinone vs. control & 24 hUTP (g/d); eGFR & $\begin{array}{l}\text { Meta- } \\
\text { Analysis }\end{array}$ & $\begin{array}{l}\text { Zhou, et al. } \\
(2020)\end{array}$ \\
\hline Tanshinone IIA & hypertensive nephropathy & 1,696 & Tanshinone IIA/ARBs vs. ARBs & eGFR & $\begin{array}{l}\text { Meta- } \\
\text { Analysis }\end{array}$ & Xu, et al. (2019) \\
\hline Berberine & $\begin{array}{l}\text { hypertensive patients with type } \\
2 \text { diabetes mellitus }\end{array}$ & 69 & control vs. berberine add-on & $\operatorname{UACR}(\mu \mathrm{g} / \mathrm{mg})$ & 2 years & Dai, et al. (2015) \\
\hline $\begin{array}{l}\text { Tripterygium } \\
\text { Glycosides }\end{array}$ & DN & 1,810 & tripterygium glycosides + ARBs vs. ARBs & $\begin{array}{l}24 \text { hUTP }(\mathrm{g} / \mathrm{d}) ; \text { UAER } \\
\text { (mg/min); SCr }\end{array}$ & $\begin{array}{l}\text { Meta- } \\
\text { Analysis }\end{array}$ & $\begin{array}{l}\text { Wu, et al. } \\
(2020 a)\end{array}$ \\
\hline Tripterygium Wilfordii & DKD (stage IV) & 1,414 & $\begin{array}{l}\text { Tripterygium Wilfordii +(ARB/ACEI) vs. (ARB/ } \\
\text { ACEI) }\end{array}$ & $\begin{array}{l}24 \text { hUTP }(g / d) ; \text { Alb }(g / \\
\text { l); TER }\end{array}$ & $\begin{array}{l}\text { Meta- } \\
\text { Analysis }\end{array}$ & $\begin{array}{l}\text { Ren, et al. } \\
(2019)\end{array}$ \\
\hline $\begin{array}{l}\text { Tripterygium } \\
\text { Preparations }\end{array}$ & CKD & 4,386 & $\begin{array}{l}\text { Tripterygium preparations vs. placebo, } \\
\text { standard care, or other immunosuppressive } \\
\text { treatment }\end{array}$ & $\begin{array}{l}\text { UPE; SCr(mg/dL); } \\
\text { CR; PR; relapse }\end{array}$ & $\begin{array}{l}\text { Meta- } \\
\text { Analysis }\end{array}$ & $\begin{array}{l}\text { Zhu, et al. } \\
\text { (2013) }\end{array}$ \\
\hline $\begin{array}{l}\text { Tripterygium } \\
\text { glycosides }\end{array}$ & DN & 70 & Tripterygium glycosides + ARBs vs. ARBs & 24 h UTP & 48 weeks & $\begin{array}{l}\text { Lengnan, et al. } \\
\text { (2020) }\end{array}$ \\
\hline Puerarin & DN(Stage III) & 669 & Puerarin + ACEl vs. ACEI & UACR (mug/min) & $\begin{array}{l}\text { Meta- } \\
\text { Analysis }\end{array}$ & $\begin{array}{l}\text { Wang, et al. } \\
(2015 a)\end{array}$ \\
\hline
\end{tabular}

24 h UTP: 24 h urinary total protein; Alb: serum albumin; ACEl: angiotensin-converting enzyme inhibitors; ARB: angiotensin receptor blockers; CKD: chronic kidney disease; CR: complete remission; DN: diabetic nephropathy; DKD: diabetic kidney disease; eGFR: estimated glomerularfiltrationrate; IgAN: IgA nephropathy; PR partial remission; RAS: renin angiotensin system; SCr: serum creatinine; TER: total effective rate; UACR: Urinary Albumin Creatinine Ratio; UAER: urinary albumin excretion rate; Tripterygium Preparations: Tripterygium glycoside tablets, Tripterygium hypoglaucum Hutch tablets, and Tripterygium granules or extracts.

1-2, with moderate proteinuria) from 26 hospitals in China. The results show that Abelmoschus manihot can effectively reduce urine protein, and no obvious adverse events were identified. In another multicenter randomized clinical trial, 1470 biopsyproven IgAN patients (proteinuria between 0.5 and $3.0 \mathrm{~g} / \mathrm{d}$ and eGFR of $>/=45 \mathrm{ml} / \mathrm{min} / 1.73 \mathrm{~m}^{2}$ were treated with either Abelmoschus manihot or losartan at 1:1, observed for 48 weeks. The results indicated that the effectiveness of Abelmoschus manihot was similar to losartan in reducing urinary proteins (Li, et al., 2020a). The eGFR was stable and did not show a significant decline in both treatment groups during the 48-weeks follow-up. The rates of adverse events did not differ between the two treatment groups (Li, et al., 2020a). Thus, Abelmoschus manihot can be used to treat patients who may not tolerate ACEI/ARB due to hypotension or other disease. As such, Abelmoschus manihot appears effective and safe in improving proteinuria and preserve renal function in patients with CKD. The long-term benefits of Abelmoschus manihot in reducing the risk of progressive renal dysfunction remain unclear and need further study. 


\section{SALVIA MILTIORRHIZA AND ITS COMPONENTS: TANSHINONE AND SALVIANOLIC ACID}

Salvia miltiorrhiza, also called "Danshen" in Chinese, is a popular Chinese herb from dried roots of $S$. miltiorrhiza Bunge, has been used for over 2,000 years for the treatment of cardiovascular diseases without obvious side effects (Yan, et al., 2018). The active ingredient of Danshen is tanshinone, which contains more than 50 compounds such as tanshinone I, tanshinone IIA, and tanshinone IIB as well as the water-soluble compounds salvianolic acid A (SAA), salvianolic acid B, and tanshinol (Xue, et al., 2019). Injection of Sodium tanshinone IIA sulfonate (STS), the extract of Danshen, has been widely used in current clinical practice in China to treat CKD in recent years.

\section{Animal Studies}

Several models of CKD were used to study the efficacy and action of mechanisms of Salvia miltiorrhiza. In the five-sixth nephrectomy model (Zhang, et al., 2019b) (Zhang, et al., 2019a) (Zhang, et al., 2018) (Wang, et al., 2015b), administration of Salvianolic acid A (SAA) and Tanshinone IIA were shown to attenuate oxidative stress and inflammation by activating the Akt/GSK-3beta/Nrf2 signaling pathway and upregulation of bone morphogenetic protein 7 (BMP-7) and Smad6 as well as inhibiting NF- $\kappa$ B and p38 MAPK and TGF- $\beta /$ Smad signaling pathways. In adriamycin (ADR)-induced minimal change disease (MCD) rat model, SAA exhibited a significant anti-proteinuria effect (Wang, et al., 2019b). In a rat model of STZ-induced diabetes, Tanshinone IIA and SAA attenuates renal damage via inhibiting oxidative stress and inflammation (Chen, et al., 2017) (Hou, et al., 2017). SAA restored glomerular endothelial permeability via AGE-RAGE-RhoA/ROCK and disturbed autophagy via AGE-RAGE-Nox4 axis (Hou, et al., 2017). Tanshinone IIA also reduced endoplasmic reticulum stress via attenuated PERK signaling activities ( $\mathrm{Xu}$, et al., 2020a). In UUO rats, Salvianolic acid B attenuates renal interstitial fibrosis by regulating the heparanase/syndecan-1 axis ( $\mathrm{Hu}$, et al., 2020), Salvianolic acid A and $\mathrm{C}$ reduced the secretion of renal inflammatory cytokines CCL5 and CXCL10 to protect renal function, improve tubular function and renal pathology (Li, et al., 2015). In adenine and potassium oxonateinduced uric acid nephropathy mice or renal ischemiareperfusion injury rats, Tanshinone IIA suppressed oxidative stress-activated MAPK pathways (Zhang, et al., 2020c), and salvianolic Acid B modulates caspase-1-mediated pyroptosis via blocking Nrf2 Pathway (Pang, et al., 2020), suppressing oxidative stress and inflammation through activation of PI3K/ Akt signaling pathway (Ma, et al., 2017b). Furthermore, tanshinone IIA attenuates contrast-induced nephropathy via enhancing Nrf2/ARE activation rats (Liang, et al., 2018). Tanshinone I protects mice from aristolochic acid I-induced kidney injury by induction of cytochrome P450 1A (Feng, et al., 2013). Salvianolic Acid B protects against fatty acidinduced renal tubular injury via inhibition of endoplasmic reticulum stress, play an important role in obesity-related kidney injury (Mai, et al., 2020). Overall, Salvia miltiorrhiza can protect against kidney disease through diverse mechanisms involved in inhibiting multiple profibrotic pathways.

\section{Clinical Studies}

Many small-clinical trials have been performed to evaluate the efficacy of Salvia miltiorrhiza in patients with CKD. A recent meta-analysis has summarized the results of Salvia miltiorrhiza (tanshinone) for CKD treatment (Zhou, et al., 2020). Twenty-one studies were reviewed in this meta-analysis, which involved 1857 patients including 954 cases from the salvia miltiorrhiza treatment group and 903 cases from the control group. It was found that Salvia miltiorrhiza could reduce urine protein levels, improve kidney function, and attenuate CKD without significant side effects. Among 21 studies included in this meta-analysis, only 2 evaluated the safety of tanshinone, tanshinone administration did not significantly the side effects.

Recently, another meta-analysis assessed the efficacy and safety of Sodium tanshinone IIA sulfonate in treatment of hypertensive nephropathy. Sixteen clinical trials involving 1,696 patients were included in this meta-analysis. It was interestingly found that a combination of Tanshinone IIA (TIIA) and angiotensin receptor blockers (ARBs) was more effective than ARB monotherapy in modulating hypertensive nephropathy (Xu, et al., 2019). This was indicated by improved eGFR and reduced urinary protein, serum creatinine, cystatin- $\mathrm{C}$, and better control in systolic blood pressure (SBP) and diastolic blood pressure (DBP) in group combined with STS plus ARBs than in ARBs alone group. Thus, it appears that STS can be used as an adjuvant agent in the management of hypertensive nephropathy. Nevertheless, all included trials in this meta-analysis report were published in Chinese, sample size for individual trials were small and the treatment course was short (2-4 weeks). To achieve more conclusive results, other large-scale, multicenter, long-term and rigorously designed RCTs should be conducted in the future.

\section{RHIZOMA COPTIDIS AND ITS COMPONENTS BERBERINE}

Rhizoma coptidis is known as "Huanglian" in Chinese. Modern pharmacological studies have demonstrated that Rhizoma coptidis and its component berberine have various pharmacological activities, including anti-inflammatory, hypoglycemic, antihypertensive, antibacterial, and other effects.

\section{Animal Studies}

The potential effects of berberine on renal interstitial fibrosis has been examined in animal models of UUO, DKD and hypertensive nephropathy. In a rat model of UUO, it was found that administration of berberine $(200 \mathrm{mg} / \mathrm{kg}$ per day) attenuated deposition of extracellular matrix, inhibited inflammation, reduced oxidative stress and suppressed TGF $\beta 1 / \mathrm{Smad} 3$ signaling pathways (Wang, et al., 2014). In a murine model of $\mathrm{DKD}$, berberine treatment was observed to reverse the disordered metabolism, podocyte damage, glomerulosclerosis, lipid accumulation, excessive generation of mitochondrial ROS, 
mitochondrial dysfunction, and deficient fatty acid oxidation through a mechanism associates with inactivation of the PGC1alpha signaling pathway (Qin, et al., 2020). Berberine could also significantly inhibited urine protein excretion, ameliorated tubulointerstitial fibrosis, protect glomerular podocytes in this model (Sun, et al., 2015, Zhang, et al., 2020b, Li and Zhang 2017, Qin, et al., 2019). The renoprotective effect of berberine is related to activating autophagy via AMPK phosphorylation (Zhang, et al., 2020b), inhibiting inflammation via suppressing TLR4/

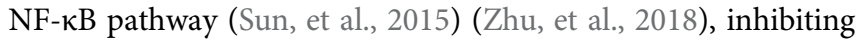
the Nrf2 pathway, and regulating the proteins expression of the matrix metalloproteinases (MMPs)/tissue inhibitors of metalloproteinases (TIMPs) (Ni, et al., 2015) and G proteincoupled receptor kinases (GRKs) (Wang, et al., 2013). In addition, berberine could delay the onset and attenuate the severity of hypertension, ameliorate hypertension-induced renal damage in spontaneously hypertensive rats. This action of berberine is associated with inhibition RAS activities and expression of some pre-inflammatory cytokines IL-6, IL-17, and IL-23 (Guo, et al., 2015b). The anti-hypertension and anti-inflammatory effects were also observed in two-kidney, one-clip (2K1C) renovascular hypertensive rats, associated with inhibition of the ROS/Erk1/2/iNOS pathway (Tian, et al., 2019).

\section{Clinical Studies}

Clinical studies have shown that berberine treatment is beneficial for hypertensive patients with T2DM (Dai, et al., 2015). In a 2years random clinical trial on 69 hypertensive patients with T2DM, whose blood pressure and fasting plasma glucose were adequately controlled prior to the study, were enrolled and randomly assigned into add-on (36 cases) and control (33 cases) groups. Berberine was orally administrated to the patients in the add-on group concomitantly with standard hypotensive and hypoglycemic treatment. Adequately control of blood pressure and glucose was observed in those two groups. In the berberine add-on group of patients, a significant reduction in UACR, urinary osteopontin and KIM1 was further observed in addition to improved renal hemodynamics, reduced renal inflammation, and oxidative stress. Therefore, berberine might be used as an alternative therapeutic strategy for the management of kidney injury. Other clinical trials are needed to investigate the efficacy of berberine in $\mathrm{CKD}$ induced by other etiologies.

\section{TRIPTERYGIUM WILFORDII AND ITS COMPONENTS: TRIPTOLIDE, TRIPTERYGIUM GLYCOSIDES}

Triptolide and tripterygium glycosides are the main bioactive constituents isolated from the Chinese herb Tripterygium wilfordii, also called as "Leigongteng." Pharmacological studies have shown that Triptolide exhibits multiple effects, including renal protective, antitumor, anti-inflammatory, immunosuppressive, cardioprotective, antiangiogenesis activities, and multiorgan toxicity effects. Triptolides have been extensively used to treat some primary and secondary kidney disease such as nephritis, minimal change disease and membranous nephropathy in humans. Its anti-fibrotic effects have been also reported in some of animal models as indicated below.

\section{Animal Studies}

In a rat model of diabetic nephropathy, tripterygium glycosides was shown to attenuate glomerulosclerosis and interstitial fibrosis and exert anti-microinflammatory effects (Wu, et al., 2017). The effect of Triptolide-elicited renoprotection in diabetic nephropathy is associated with regulating $\mathrm{T}$ cell balance and reducing macrophage infiltration to the kidney (Guo, et al., 2016), attenuates renal tubular EMT (Xue, et al., 2018), prevents extracellular matrix accumulation by targeting microRNA-137/ Notch1 (Han, et al., 2018) and miR-141-3p/PTEN/Akt/mTOR pathway (Li, et al., 2017b). In a rat UUO model, treatment with Triptolide also decreased interstitial collagen deposition, inhibited renal interstitial fibroblast activation and suppressed production of proinflammtory and profibrogenetic factors, including TGF- $\beta 1$, connective tissue growth factor (CTGF), MCP1 and osteopontin (Yuan, et al., 2011). Additionally, triptolide administration significantly delayed disease progression and improved renal function in an adult rat model of polycystic kidney disease through inhibiting the JAK2-STAT3 pathway (Jing, et al., 2018), attenuates renal damage by limiting inflammatory responses in DOCA-salt hypertension (Zhang, et al., 2020a), and inhibits podocyte apoptosis by targeting IL4 to alleviate kidney injury in FSGS rats (Li, et al., 2020c).

\section{Clinical Studies}

Two meta-analyses (Ren, et al., 2019; Wu, et al., 2020a) evaluated the clinical efficacy and safety of Tripterygium wilfordii/tripterygium glycosides combined with ARB/ACEI in the treatment of stage IV DKD. In the controlled trial (RCT), 1414 participants (Ren, et al., 2019) were evaluated in detail, and another meta-analysis included 23 studies, including a total of $1810 \mathrm{DN}$ patients (Wu, et al., 2020a). Tripterygium wilfordii/tripterygium glycosides combined with ARB/ACEI significantly improved 24-h urinary total protein (24 h-UTP), urinary UAER, SCr, and albumin more than did ARB/ARB alone. Some minor side effects such as abnormal liver function tests were observed in the combined treatment group, with the risk of adverse reactions increased by $8 \%$. Moreover, a prospective, randomized controlled trial for assessing the efficacy of tripterygium wilfordii in Stage IV-DN is still in progress (Lengnan, et al., 2020).

A systematic review and meta-analysis of Tripterygium wilfordii polyglycosides in the treatment of CKD indicate that among 75 trials that included 4,386 participants, treatment with tripterygium polyglycoside preparation reduces proteinuria, lowers serum creatinine, improves the complete remission rate by $56 \%$, improves the complete or partial remission rate by $24 \%$, and reduces the relapse rate by 58\% (Zhu, et al., 2013). Tripterygium polyglycoside preparation group also increased liver function abnormalities and menstrual changes (Zhu, et al., 2013). 


\section{KUDZU ROOT PUERARIA AND ITS COMPONENT PUERARIN}

Puerarin is natural flavonoid extracted from the Chinese medical herb Radix puerariae, also called as "Gegen". Many studies have demonstrated that puerarin has a renoprotective effect in animal model of AKI induced by various nephrotoxicants such as cisplatin (Wu, et al., 2020b) (Ma, et al., 2017a), methotrexate (Liu, et al., 2018), lead (Liu, et al., 2012) and carbon tetrachloride. Recently, the effect of puerarin on CKD and mechanism involved have also been examined in animal models of UUO and DA.

\section{Animal Studies}

Puerarin treatment attenuates renal tubulointerstitial fibrosis in a murine model of UUO, as evidenced by decreased the accumulation of ECM and reduced renal tubule damage. Mechanistically, puerarin inhibited renal epithelial cell apoptosis, reduced expression of NOX4 and inhibited phosphorylation of phosphorylation of p38, ERK, and JNK, three MAPK pathways associated with renal fibrosis (Zhou, et al., 2017). In a murine model of diabetic nephropathy, puerarin also exhibits a potent renoprotective and anti-fibrotic effect through a mechanism associated with suppression of NOX4 and miRNA-140-5p expression (Li, et al., 2017a; Xu, et al., 2020c), promotion of podocyte autophagy (Li, et al., 2020b), downregulation of MMP9 (Zhong, et al., 2014), and activation of the PERK/eIF2a/ATF4 signaling pathway (Xu, et al., 2020b). In addition, puerarin also reduces the contents and expression of advanced glycation end products in the diabetic kidney (Shen, et al., 2009) and restores the expression of nephrin by inhibiting the expression of HIF-1alpha and VEGF (Shukla, et al., 2017).

\section{Clinical Studies}

A meta-analysis has assessed the beneficial and harmful effects of puerarin plus ACEI compared with ACEI alone for the treatment of individuals with stage III DN. Ten RCTs involving 669 participants were included in this meta-analysis. All trials were conducted in China and published in Chinese. Treatment of DN with puerarin plus ACEI significantly decreased the urinary albumin excretion rate (UAER) but had no effect on 24-hUTP. One trial reported abdominal discomfort and nausea (2 cases) in the treatment group. Although these studies suggest that puerarin can reduce proteinuria of individuals with stage III DN (Wang, et al., 2015a). Further clinical trials with more samples and multiple centers should be conducted to verify the beneficial results for DN.

\section{COMMON MECHANISMS OF CHINESE HERBAL MEDICINES IN TREATING KIDNEY DISEASE}

Combining the content of this article and previous literature review (Zhao, et al., 2020; Zhong, et al., 2013), we identify that eight Chinese herbal medicine have anti-inflammatory, antioxidant, anti-apoptotic effects, reducing extracellular matrix deposition, and anti-fibrosis. Among them, Salvia miltiorrhiza, Rhizoma coptidis, Abelmoschus manihot can interrupt almost all the processes leading to renal fibrogenesis. A summary of the common mechanisms of these Chinese herbal medicines in the treatment of kidney diseases is shown in Figure 1.

\section{CONCLUSION AND PERSPECTIVE}

Although preclinical animal studies often indicate therapeutic benefits of Chinese herbal medicine in models of CKD, convincing evidence for or against Chinese herbal medicine for patients with CKD is limited. Here we summerized recent advances on the therapeutic effect of five Chinese herbal compounds, either single-herbal or monomer, on CKD. Their anti-fibrotic effects are involved in the regulation of immunity, alteration of hemodynamic changes, anti-oxidative stress and fibrosis. Clearly, more research is needed to identify the active ingredients of herbal medicines effective for the treatment of $\mathrm{CKD}$ and the mechanism of action involved. Determination of a monomer with definite curative effect and mechanism of action, and optimization of the formulation of TCM through modern scientific research will further improve and confirm the clinical curative effect. Below are several issues that should be considered.

First, clinical efficacy research should be strengthened. Although some single Chinese medicines or monomers have conducted RCT studies and meta-analysis, such as "Huangkui" capsules, most others have only small samples of clinical controlled observations, such as berberine. Therefore, a largesample, long-period RCT studies should be initiated on the basis of physicians' personal clinical experience and small-sample clinical observations to confirm the clinical efficacy of TCM against renal fibrosis.

Second, mechanism study should be focused on the most critical action of mechanism in a given drug. For example, tanshinone IIA, berberine, and triptolide all have antiinflammatory and anti-oxidant actions, but it remain unclear which herb is stronger in those actions. Puerarin has antiinflammatory and antioxidant effects, regulating podocyte autophagy, tubular epithelial cell autophagy and apoptosis. It is unknown which is the most critical action of puerarin against renal fibrosis. A recent study (Zhong, et al., 2019) showing that arctigenin attenuates diabetic kidney disease through the activation of PP2A in podocytes gives us a good example and enlightenment to pursue in depth understanding of modern pharmacology of Chinese herbal medicine.

Third, the role and action of mechanism of monomer compounds need to be explored in depth. The research on the combination of Chinese medicine monomers is worth of exploration in the future. Salvia miltiorrhiza and Rhizoma coptidis often appear in one prescription of Chinese herbal medicine to treat CKD such as Shenshuaining Capsule (Cui, et al., 2016), Yishen Zhishuai Granules and Shenshuai II Recipe (Wang, et al., 2019a). According to the classic theory of Chinese traditional Medicine, Rhizoma coptidis is capable of clearing heat and dampness, purging fire and detoxification, as well as inducing Qi, whereas Salvia miltiorrhiza has an ability to activate blood and remove stasis as well as cool blood and detoxify. The combination of these two herbs can act in concert to eliminate 


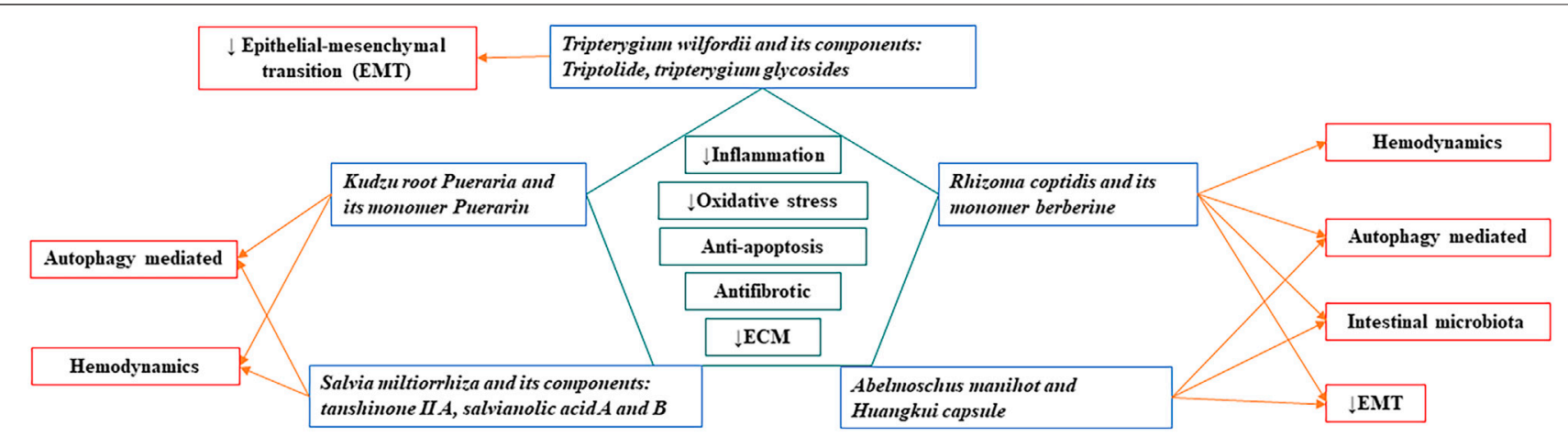

FIGURE 1 | Common mechanisms of Chinese herbal medicines in treating kidney disease.

the most common pathogenic syndromes of CKD, such as dampness, heat, blood stasis, and toxicity. Future research is necessary to explore the synergistic effect and mechanism of berberine and salvianolic acid on renal fibrosis in order to further elucidate the clinical significance and mechanism of action of Salvia miltiorrhiza and Rhizoma coptidis.

Fourth, the systems approach is necessary for exploring the synergistic effects of TCM in kidney disease. The systems approach in TCM is a methodology that combines computational and experimental tools to discover novel therapeutic agents, identify their candidate targets, and understand their therapeutic mechanisms. Traditional medicine often is a mixed formula composing of several types of herbs, and a formula with mixed herbs usually works better than single ones. However, the mechanism of action of individual herbs and their synergistic effects in a formula are frequently unknown, thus, the systems approach should be utilized to analyze the interactions among compounds in order to determine the synergistic effects of TCM in a given formula. To achieve this goal, we need to 1) identify the active ingredients from traditional medicine mixtures using modern technologies such as gas chromatography-mass spectrometr, 2) identify targets of active ingredients by developing predictive network models and analyzing the complex interactions among herbs, compounds, herb-target, and compound-target networks. 3) determine the biological activity and toxicity profile of

\section{REFERENCES}

Cai, H. D., Su, S. L., Qian, D. W., Guo, S., Tao, W. W., Cong, X. D., et al. (2017). Renal Protective Effect and Action Mechanism of Huangkui Capsule and its Main Five Flavonoids. J. Ethnopharmacol 206, 152-159. doi:10.1016/ j.jep.2017.02.046

Chen, X., Wu, R., Kong, Y., Yang, Y., Gao, Y., Sun, D., et al. (2017). Tanshinone IIA Attenuates Renal Damage in STZ-Induced Diabetic Rats via Inhibiting Oxidative Stress and Inflammation. Oncotarget 8, 31915-31922. doi:10.18632/oncotarget.16651

Cui, R. Z., Xie, Y. M., Liao, X., and Wang, J. D. (2016). Shenshuaining Capsules as Adjuvant Treatment for Chronic Renal Failure: Systematic Review and MetaAnalysis of Randomized Controlled Trials]: Systematic Review and Meta- individual active compounds and multiple ingredients in diverse combinations using both in vitro techniques and animal models of kidney disease, 4) elucidate the action of mechanism of each active compounds and their synergistic effects by using advanced systems approaches such as mass spectrometry and affymetrix microarrays for gene expression analysis, and 5) conduct clinical trial to assess the therapeutic effect of single active compounds and multiple ingredients with different combinations.

\section{AUTHOR CONTRIBUTIONS}

MS and CY drafted the manuscript, SZ and GB edited it. All the authors approved it for publication.

\section{FUNDING}

This project is supported by the National Natural Science Foundation of China (2020-81973807 to MS; 81670623 and 81830021 to SZ); State Outstanding Talents of Traditional Chinese Medicine Project of National Administration of Traditional Chinese Medicine (NATCM:2017-124 to MS); National key R\&D Program of China (2018YFA0108802 to SZ), and US National Institutes of Health (2R01DK08506505A1 to SZ).

Analysis of Randomized Controlled Trials. Zhongguo Zhong Yao Za Zhi 41, 2149-2161. doi:10.4268/cjcmm20161128

Dai, P., Wang, J., Lin, L., Zhang, Y., and Wang, Z. (2015). Renoprotective Effects of Berberine as Adjuvant Therapy for Hypertensive Patients with Type 2 Diabetes Mellitus: Evaluation via Biochemical Markers and Color Doppler Ultrasonography. Exp. Ther. Med. 10, 869-876. doi:10.3892/etm.2015.2585

Feng, C., Xie, X., Wu, M., Li, C., Gao, M., Liu, M., et al. (2013). Tanshinone I Protects Mice from Aristolochic Acid I-Induced Kidney Injury by Induction of CYP1A. Environ. Toxicol. Pharmacol. 36, 850-857. doi:10.1016/j.etap.2013.07.017

Ge, J., Miao, J. J., Sun, X. Y., and Yu, J. Y. (2016). Huangkui Capsule, an Extract from Abelmoschus Manihot (L.) Medic, Improves Diabetic Nephropathy via Activating Peroxisome Proliferator-Activated Receptor (PPAR)- $\alpha / \gamma$ and Attenuating Endoplasmic Reticulum Stress in Rats. J. Ethnopharmacol 189, 238-249. doi:10.1016/j.jep.2016.05.033 
Glassock, R. J., Warnock, D. G., and Delanaye, P. (2017). The Global burden of Chronic Kidney Disease: Estimates, Variability and Pitfalls. Nat. Rev. Nephrol. 13, 104-114. doi:10.1038/nrneph.2016.163

Gu, L., Hong, F., Fan, K., Zhao, L., Zhang, C., Yu, B., et al. (2020). Integrated Network Pharmacology Analysis and Pharmacological Evaluation to Explore the Active Components and Mechanism of Abelmoschus Manihot (L.) Medik. On Renal Fibrosis. Drug Des. Devel Ther. 14, 4053-4067. doi:10.2147/ DDDT.S264898

Guo, H., Pan, C., Chang, B., Wu, X., Guo, J., Zhou, Y., et al. (2016). Triptolide Improves Diabetic Nephropathy by Regulating Th Cell Balance and Macrophage Infiltration in Rat Models of Diabetic Nephropathy. Exp. Clin. Endocrinol. Diabetes 124, 389-398. doi:10.1055/s-0042-106083

Guo, J. M., Lu, Y. W., Shang, E. X., Li, T., Liu, Y., Duan, J. A., et al. (2015a). Metabolite Identification Strategy of Non-targeted Metabolomics and its Application for the Identification of Components in Chinese Multicomponent Medicine Abelmoschus Manihot L. Phytomedicine 22, 579-587. doi:10.1016/j.phymed.2015.02.002

Guo, Z., Sun, H., Zhang, H., and Zhang, Y. (2015b). Anti-hypertensive and Renoprotective Effects of Berberine in Spontaneously Hypertensive Rats. Clin. Exp. Hypertens. 37, 332-339. doi:10.3109/10641963.2014.972560

Han, F., Wang, S., Chang, Y., Li, C., Yang, J., Han, Z., et al. (2018). Triptolide Prevents Extracellular Matrix Accumulation in Experimental Diabetic Kidney Disease by Targeting microRNA-137/Notch1 Pathway. J. Cel Physiol 233, 2225-2237. doi:10.1002/jcp.26092

Hou, B., Qiang, G., Zhao, Y., Yang, X., Chen, X., Yan, Y., et al. (2017). Salvianolic Acid A Protects against Diabetic Nephropathy through Ameliorating Glomerular Endothelial Dysfunction via Inhibiting AGE-RAGE Signaling. Cell Physiol Biochem 44, 2378-2394. doi:10.1159/000486154

Hu, Y., Wang, M., Pan, Y., Li, Q., and Xu, L. (2020). Salvianolic Acid B Attenuates Renal Interstitial Fibrosis by Regulating the HPSE/SDC1 axis. Mol. Med. Rep. 22, 1325-1334. doi:10.3892/mmr.2020.11229

Jing, Y., Wu, M., Zhang, D., Chen, D., Yang, M., Mei, S., et al. (2018). Triptolide Delays Disease Progression in an Adult Rat Model of Polycystic Kidney Disease through the JAK2-STAT3 Pathway. Am. J. Physiol. Ren. Physiol 315, F479-F486. doi:10.1152/ajprenal.00329.2017

Kim, H., Dusabimana, T., Kim, S. R., Je, J., Jeong, K., Kang, M. C., et al. (2018). Supplementation of Abelmoschus Manihot Ameliorates Diabetic Nephropathy and Hepatic Steatosis by Activating Autophagy in Mice. Nutrients 10. doi:10.3390/nu10111703

Lengnan, X., Ban, Z., Haitao, W., Lili, L., Aiqun, C., Huan, W., et al. (2020). Tripterygium Wilfordii Hook F Treatment for Stage IV Diabetic Nephropathy: Protocol for a Prospective, Randomized Controlled Trial. Biomed. Res. Int. 2020, 9181037. doi:10.1155/2020/9181037

Li, J., Gu, T., Fu, X., and Zhao, R. (2015). Effect of Salvianolic Acid A and C Compatibility on Inflammatory Cytokines in Rats with Unilateral Ureteral Obstruction. J. Tradit Chin. Med. 35, 564-570. doi:10.1016/s0254-6272(15) 30140-0

Li, P., Lin, H., Ni, Z., Zhan, Y., He, Y., Yang, H., et al. (2020a). Efficacy and Safety of Abelmoschus Manihot for IgA Nephropathy: A Multicenter Randomized Clinical Trial. Phytomedicine 76, 153231. doi:10.1016/j.phymed.2020.153231

Li, W., He, W., Xia, P., Sun, W., Shi, M., Zhou, Y., et al. (2019). Total Extracts of Abelmoschus Manihot L. Attenuates Adriamycin-Induced Renal Tubule Injury via Suppression of ROS-Erk1/2-Mediated NLRP3 Inflammasome Activation. Front. Pharmacol. 10, 567. doi:10.3389/fphar.2019.00567

Li, X., Cai, W., Lee, K., Liu, B., Deng, Y., Chen, Y., et al. (2017a). Puerarin Attenuates Diabetic Kidney Injury through the Suppression of NOX4 Expression in Podocytes. Sci. Rep. 7, 14603. doi:10.1038/s41598-017-14906-8

Li, X., Zhu, Q., Zheng, R., Yan, J., Wei, M., Fan, Y., et al. (2020b). Puerarin Attenuates Diabetic Nephropathy by Promoting Autophagy in Podocytes. Front. Physiol. 11, 73. doi:10.3389/fphys.2020.00073

Li, X. Y., Wang, S. S., Han, Z., Han, F., Chang, Y. P., Yang, Y., et al. (2017b). Triptolide Restores Autophagy to Alleviate Diabetic Renal Fibrosis through the miR-141-3p/PTEN/Akt/mTOR Pathway. Mol. Ther. Nucleic Acids 9, 48-56. doi:10.1016/j.omtn.2017.08.011

Li, Y., Jiang, X., Song, L., Yang, M., and Pan, J. (2020c). Anti-apoptosis Mechanism of Triptolide Based on Network Pharmacology in Focal Segmental Glomerulosclerosis Rats. Biosci. Rep. 40. doi:10.1042/BSR20192920
Li, Z., and Zhang, W. (2017). Protective Effect of Berberine on Renal Fibrosis Caused by Diabetic Nephropathy. Mol. Med. Rep. 16, 1055-1062. doi:10.3892/ mmr.2017.6707

Liang, R., Zhao, Q., Jian, G., Cheng, D., Wang, N., Zhang, G., et al. (2018). Tanshinone IIA Attenuates Contrast-Induced Nephropathy via Nrf2 Activation in Rats. Cel Physiol Biochem 46, 2616-2623. doi:10.1159/000489688

Liao, Z., Zhang, J., Wang, J., Yan, T., Xu, F., Wu, B., et al. (2019). The Anti-nephritic Activity of a Polysaccharide from Okra (Abelmoschus Esculentus (L.) Moench) via Modulation of AMPK-Sirt1-PGC-1a Signaling axis Mediated Antioxidative in Type 2 Diabetes Model Mice. Int. J. Biol. Macromol 140, 568-576. doi:10.1016/j.ijbiomac.2019.08.149

Lin, M. Y., Chiu, Y. W., Chang, J. S., Lin, H. L., Lee, C. T., Chiu, G. F., et al. (2015). Association of Prescribed Chinese Herbal Medicine Use with Risk of End-Stage Renal Disease in Patients with Chronic Kidney Disease. Kidney Int. 88, 1365-1373. doi:10.1038/ki.2015.226

Liu, C. M., Ma, J. Q., and Sun, Y. Z. (2012). Puerarin Protects Rat Kidney from leadinduced Apoptosis by Modulating the PI3K/Akt/eNOS Pathway. Toxicol. Appl. Pharmacol. 258, 330-342. doi:10.1016/j.taap.2011.11.015

Liu, Q., Liu, Z., Huo, X., Wang, C., Meng, Q., Sun, H., et al. (2018). Puerarin Improves Methotrexate-Induced Renal Damage by Up-Regulating Renal Expression of Oat1 and Oat3 In Vivo and In Vitro. Biomed. Pharmacother. 103, 915-922. doi:10.1016/j.biopha.2018.04.122

Liu, S., Ye, L., Tao, J., Ge, C., Huang, L., and Yu, J. (2017). Total Flavones of Abelmoschus Manihot Improve Diabetic Nephropathy by Inhibiting the iRhom2/TACE Signalling Pathway Activity in Rats. Pharm. Biol. 56, 1-11. doi:10.1080/13880209.2017.1412467

Liu, Y. (2011). Cellular and Molecular Mechanisms of Renal Fibrosis. Nat. Rev. Nephrol. 7, 684-696. doi:10.1038/nrneph.2011.149

Ma, X., Yan, L., Zhu, Q., and Shao, F. (2017a). Puerarin Attenuates CisplatinInduced Rat Nephrotoxicity: The Involvement of TLR4/NF-Kb Signaling Pathway. PLoS One 12, e0171612. doi:10.1371/journal.pone.0171612

Ma, Z. G., Xia, H. Q., Cui, S. L., and Yu, J. (2017b). Attenuation of Renal Ischemic Reperfusion Injury by Salvianolic Acid B via Suppressing Oxidative Stress and Inflammation through PI3K/Akt Signaling Pathway. Braz. J. Med. Biol. Res. 50, e5954. doi:10.1590/1414-431X20175954

Mai, X., Yin, X., Chen, P., and Zhang, M. (2020). Salvianolic Acid B Protects against Fatty Acid-Induced Renal Tubular Injury via Inhibition of Endoplasmic Reticulum Stress. Front. Pharmacol. 11, 574229. doi:10.3389/fphar.2020.574229

Meng, X. M., Nikolic-Paterson, D. J., and Lan, H. Y. (2016). TGF- $\beta$ : the Master Regulator of Fibrosis. Nat. Rev. Nephrol. 12, 325-338. doi:10.1038/ nrneph.2016.48

Ni, W. J., Ding, H. H., Zhou, H., Qiu, Y. Y., and Tang, L. Q. (2015). Renoprotective Effects of Berberine through Regulation of the MMPs/TIMPs System in Streptozocin-Induced Diabetic Nephropathy in Rats. Eur. J. Pharmacol. 764, 448-456. doi:10.1016/j.ejphar.2015.07.040

Pang, Y., Zhang, P. C., Lu, R. R., Li, H. L., Li, J. C., Fu, H. X., et al. (2020). AndradeOliveira Salvianolic Acid B Modulates Caspase-1-Mediated Pyroptosis in Renal Ischemia-Reperfusion Injury via Nrf2 Pathway. Front. Pharmacol. 11, 541426. doi:10.3389/fphar.2020.541426

Peng, C. H., Chyau, C. C., Wang, C. J., Lin, H. T., Huang, C. N., and Ker, Y. B. (2016). Abelmoschus Esculentus Fractions Potently Inhibited the Pathogenic Targets Associated with Diabetic Renal Epithelial to Mesenchymal Transition. Food Funct. 7, 728-740. doi:10.1039/c5fo01214g

Qin, X., Jiang, M., Zhao, Y., Gong, J., Su, H., Yuan, F., et al. (2020). Berberine Protects against Diabetic Kidney Disease via Promoting PGC-1 $\alpha$-Regulated Mitochondrial Energy Homeostasis. Br. J. Pharmacol. 177, 3646-3661. doi:10.1111/bph.14935

Qin, X., Zhao, Y., Gong, J., Huang, W., Su, H., Yuan, F., et al. (2019). Berberine Protects Glomerular Podocytes via Inhibiting Drp1-Mediated Mitochondrial Fission and Dysfunction. Theranostics 9, 1698-1713. doi:10.7150/thno.30640

Ren, D., Zuo, C., and Xu, G. (2019). Clinical Efficacy and Safety of Tripterygium Wilfordii Hook in the Treatment of Diabetic Kidney Disease Stage IV: A MetaAnalysis of Randomized Controlled Trials. Medicine (Baltimore) 98, e14604. doi:10.1097/MD.0000000000014604

Ruiz-Ortega, M., Rayego-Mateos, S., Lamas, S., Ortiz, A., and Rodrigues-Diez, R. R. (2020). Targeting the Progression of Chronic Kidney Disease. Nat. Rev. Nephrol. 16, 269-288. doi:10.1038/s41581-019-0248-y 
Shen, J. G., Yao, M. F., Chen, X. C., Feng, Y. F., Ye, Y. H., and Tong, Z. H. (2009). Effects of Puerarin on Receptor for Advanced Glycation End Products in Nephridial Tissue of Streptozotocin-Induced Diabetic Rats. Mol. Biol. Rep. 36, 2229-2233. doi:10.1007/s11033-008-9438-6

Shi, L., Feng, L., Zhang, M., Li, X., Yang, Y., Zhang, Y., et al. (2019). Abelmoschus Manihot for Diabetic Nephropathy: A Systematic Review and Meta-Analysis. Evid. Based Complement. Alternat Med. 2019, 9679234. doi:10.1155/2019/ 9679234

Shukla, R., Pandey, N., Banerjee, S., and Tripathi, Y. B. (2017). Effect of Extract of Pueraria Tuberosa on Expression of Hypoxia Inducible Factor-1 $\alpha$ and Vascular Endothelial Growth Factor in Kidney of Diabetic Rats. Biomed. Pharmacother. 93, 276-285. doi:10.1016/j.biopha.2017.06.045

Sun, S. F., Zhao, T. T., Zhang, H. J., Huang, X. R., Zhang, W. K., Zhang, L., et al. (2015). Renoprotective Effect of Berberine on Type 2 Diabetic Nephropathy in Rats. Clin. Exp. Pharmacol. Physiol. 42, 662-670. doi:10.1111/14401681.12402

Tian, H., Kang, Y. M., Gao, H. L., Shi, X. L., Fu, L. Y., Li, Y., et al. (2019). Chronic Infusion of Berberine into the Hypothalamic Paraventricular Nucleus Attenuates Hypertension and Sympathoexcitation via the ROS/Erk1/2/iNOS Pathway. Phytomedicine 52, 216-224. doi:10.1016/j.phymed.2018.09.206

Tu, Y., Fang, Q. J., Sun, W., Liu, B. H., Liu, Y. L., Wu, W., et al. (2020). Total Flavones of Abelmoschus Manihot Remodels Gut Microbiota and Inhibits Microinflammation in Chronic Renal Failure Progression by Targeting Autophagy-Mediated Macrophage Polarization. Front. Pharmacol. 11, 566611. doi:10.3389/fphar.2020.566611

Tu, Y., Sun, W., Wan, Y. G., Che, X. Y., Pu, H. P., Yin, X. J., et al. (2013). Huangkui Capsule, an Extract from Abelmoschus Manihot (L.) Medic, Ameliorates Adriamycin-Induced Renal Inflammation and Glomerular Injury via Inhibiting p38MAPK Signaling Pathway Activity in Rats. J. Ethnopharmacol 147, 311-320. doi:10.1016/j.jep.2013.03.006

Wang, B., Chen, S., Yan, X., Li, M., Li, D., Lv, P., et al. (2015a). The Therapeutic Effect and Possible Harm of Puerarin for Treatment of Stage III Diabetic Nephropathy: a Meta-Analysis. Altern. Ther. Health Med. 21, 36-44.

Wang, D. T., Huang, R. H., Cheng, X., Zhang, Z. H., Yang, Y. J., and Lin, X. (2015b). Tanshinone IIA Attenuates Renal Fibrosis and Inflammation via Altering Expression of TGF- $\beta /$ Smad and NF-Kb Signaling Pathway in $5 / 6$ Nephrectomized Rats. Int. Immunopharmacol 26, 4-12. doi:10.1016/ j.intimp.2015.02.027

Wang, F. L., Tang, L. Q., Yang, F., Zhu, L. N., Cai, M., and Wei, W. (2013). Renoprotective Effects of Berberine and its Possible Molecular Mechanisms in Combination of High-Fat Diet and Low-Dose Streptozotocin-Induced Diabetic Rats. Mol. Biol. Rep. 40, 2405-2418. doi:10.1007/s11033-0122321-5

Wang, F. M., Yang, Y. J., Ma, L. L., Tian, X. J., and He, Y. Q. (2014). Berberine Ameliorates Renal Interstitial Fibrosis Induced by Unilateral Ureteral Obstruction in Rats. Nephrology (Carlton) 19, 542-551. doi:10.1111/nep.12271

Wang, M., Yang, L., Yang, J., and Wang, C. (2019a). Shen Shuai IIRecipe Attenuates Renal Injury and Fibrosis in Chronic Kidney Disease by Regulating NLRP3 Inflammasome and Sirt1/Smad3 Deacetylation Pathway. BMC Complement. Altern. Med. 19, 107. doi:10.1186/s12906-019-2524-6

Wang, X., Qi, D., Fu, F., Li, X., Liu, Y., Ji, K., et al. (2019b). Therapeutic and Antiproteinuric Effects of Salvianolic Acid A in Combined with Low-Dose Prednisone in Minimal Change Disease Rats: Involvement of PPAR $\gamma /$ Angptl4 and Nrf2/HO-1 Pathways. Eur. J. Pharmacol. 858, 172342. doi:10.1016/ j.ejphar.2019.04.023

Wu, W., Yang, J. J., Yang, H. M., Huang, M. M., Fang, Q. J., Shi, G., et al. (2017). Multi-glycoside of Tripterygium Wilfordii Hook. F. Attenuates Glomerulosclerosis in a Rat Model of Diabetic Nephropathy by Exerting Anti-microinflammatory Effects without Affecting Hyperglycemia. Int. J. Mol. Med. 40, 721-730. doi:10.3892/ijmm.2017.3068

Wu, X., Huang, Y., Zhang, Y., He, C., Zhao, Y., Wang, L., et al. (2020a). Efficacy of Tripterygium Glycosides Combined with ARB on Diabetic Nephropathy: a Meta-Analysis. Biosci. Rep. 40. doi:10.1042/BSR20202391

Wu, Z., Li, C., Li, Q., Li, J., and Lu, X. (2020b). Puerarin Alleviates CisplatinInduced Acute Renal Damage and Upregulates microRNA-31-Related Signaling. Exp. Ther. Med. 20, 3122-3129. doi:10.3892/etm.2020.9081

Xu, J., Zhang, C., Shi, X., Li, J., Liu, M., Jiang, W., et al. (2019). Efficacy and Safety of Sodium Tanshinone IIA Sulfonate Injection on Hypertensive Nephropathy: A
Systematic Review and Meta-Analysis. Front. Pharmacol. 10, 1542. doi:10.3389/ fphar.2019.01542

Xu, S., He, L., Ding, K., Zhang, L., Xu, X., Wang, S., et al. (2020a). Tanshinone IIA Ameliorates Streptozotocin-Induced Diabetic Nephropathy, Partly by Attenuating PERK Pathway-Induced Fibrosis. Drug Des. Devel Ther. 14, 5773-5782. doi:10.2147/DDDT.S257734

Xu, X., Chen, B., Huang, Q., Wu, Y., and Liang, T. (2020b). The Effects of Puerarin on Autophagy through Regulating of the PERK/eIF2a/ATF4 Signaling Pathway Influences Renal Function in Diabetic Nephropathy. Diabetes Metab. Syndr. Obes. 13, 2583-2592. doi:10.2147/DMSO.S256457

Xu, Y., Xiong, Y., Xu, C., and Xu, C. (2020c). Standard Puerarin Prevents Diabetic Renal Damage by Inhibiting miRNA-140-5p Expression. Diabetes Metab. Syndr. Obes. 13, 3947-3958. doi:10.2147/dmso.S273952

Xue, J., Jin, X., Wan, X., Yin, X., Fang, M., Liu, T., et al. (2019). Effects and Mechanism of Tanshinone II A in Proliferation, Apoptosis, and Migration of Human Colon Cancer Cells. Med. Sci. Monit. 25, 4793-4800. doi:10.12659/ msm.914446

Xue, M., Cheng, Y., Han, F., Chang, Y., Yang, Y., Li, X., et al. (2018). Triptolide Attenuates Renal Tubular Epithelial-Mesenchymal Transition via the MiR188-5p-Mediated PI3K/AKT Pathway in Diabetic Kidney Disease. Int. J. Biol. Sci. 14, 1545-1557. doi:10.7150/ijbs.24032

Yan, S. H., Zhao, N. W., Geng, Z. R., Shen, J. Y., Liu, F. M., Yan, D., et al. (2018), Modulations of Keap1-Nrf2 Signaling axis by TIIA Ameliorated the Oxidative Stress-Induced Myocardial Apoptosis. Free Radic. Biol. Med. 115, 191-201. doi:10.1016/j.freeradbiomed.2017.12.001

Yuan, X. P., He, X. S., Wang, C. X., Liu, L. S., and Fu, Q. (2011). Triptolide Attenuates Renal Interstitial Fibrosis in Rats with Unilateral Ureteral Obstruction. Nephrology (Carlton) 16, 200-210. doi:10.1111/j.14401797.2010.01359.x

Zhang, G., Cui, G., Tong, S., and Cao, Q. (2019a). Salvianolic Acid A Alleviates the Renal Damage in Rats with Chronic Renal Failurel. Acta Cir Bras 34, e201900204. doi:10.1590/s0102-8650201900204

Zhang, H. F., Wang, J. H., Wang, Y. L., Gao, C., Gu, Y. T., Huang, J., et al. (2019b). Salvianolic Acid A Protects the Kidney against Oxidative Stress by Activating the Akt/GSK-3 $3 /$ Nrf2 Signaling Pathway and Inhibiting the NF-Kb Signaling Pathway in 5/6 Nephrectomized Rats. Oxid Med. Cel Longev 2019, 2853534. doi:10.1155/2019/2853534

Zhang, H. F., Wang, Y. L., Gao, C., Gu, Y. T., Huang, J., Wang, J. H., et al. (2018). Salvianolic Acid A Attenuates Kidney Injury and Inflammation by Inhibiting NF-Kb and P38 MAPK Signaling Pathways in 5/6 Nephrectomized Rats. Acta Pharmacol. Sin 39, 1855-1864. doi:10.1038/s41401-018-0026-6

Zhang, J., Zhu, M., Zhang, S., Xie, S., Gao, Y., and Wang, Y. (2020a). Triptolide Attenuates Renal Damage by Limiting Inflammatory Responses in DOCA-Salt Hypertension. Int. Immunopharmacol 89, 107035. doi:10.1016/ j.intimp.2020.107035

Zhang, L., Li, P., Xing, C. Y., Zhao, J. Y., He, Y. N., Wang, J. Q., et al. (2014). Efficacy and Safety of Abelmoschus Manihot for Primary Glomerular Disease: a Prospective, Multicenter Randomized Controlled Clinical Trial. Am. J. Kidney Dis. 64, 57-65. doi:10.1053/j.ajkd.2014.01.431

Zhang, M., Zhang, Y., Xiao, D., Zhang, J., Wang, X., Guan, F., et al. (2020b). Highly Bioavailable Berberine Formulation Ameliorates Diabetic Nephropathy through the Inhibition of Glomerular Mesangial Matrix Expansion and the Activation of Autophagy. Eur. J. Pharmacol. 873, 172955. doi:10.1016/ j.ejphar.2020.172955

Zhang, X. W., Zhou, M., An, L., Zhang, P., Li, P., and Chen, J. (2020c). Lipophilic Extract and Tanshinone IIA Derived from Salvia Miltiorrhiza Attenuate Uric Acid Nephropathy through Suppressing Oxidative Stress-Activated MAPK Pathways. Am. J. Chin. Med. 48, 1455-1473. doi:10.1142/S0192415X20500718

Zhao, M., Yu, Y., Wang, R., Chang, M., Ma, S., Qu, H., et al. (2020). Mechanisms and Efficacy of Chinese Herbal Medicines in Chronic Kidney Disease. Front. Pharmacol. 11, 619201. doi:10.3389/fphar.2020.619201

Zhong, Y., Deng, Y., Chen, Y., Chuang, P. Y., and Cijiang He, J. (2013). Therapeutic Use of Traditional Chinese Herbal Medications for Chronic Kidney Diseases. Kidney Int. 84, 1108-1118. doi:10.1038/ki.2013.276

Zhong, Y., Lee, K., Deng, Y., Ma, Y., Chen, Y., Li, X., et al. (2019). Arctigenin Attenuates Diabetic Kidney Disease through the Activation of PP2A in Podocytes. Nat. Commun. 10, 4523. doi:10.1038/s41467-019-12433-w 
Zhong, Y., Zhang, X., Cai, X., Wang, K., Chen, Y., and Deng, Y. (2014). Puerarin Attenuated Early Diabetic Kidney Injury through Down-Regulation of Matrix Metalloproteinase 9 in Streptozotocin-Induced Diabetic Rats. PLoS One 9, e85690. doi:10.1371/journal.pone.0085690

Zhou, L., An, X. F., Teng, S. C., Liu, J. S., Shang, W. B., Zhang, A. H., et al. (2012). Pretreatment with the Total Flavone Glycosides of Flos Abelmoschus Manihot and Hyperoside Prevents Glomerular Podocyte Apoptosis in StreptozotocinInduced Diabetic Nephropathy. J. Med. Food 15, 461-468. doi:10.1089/ jmf.2011.1921

Zhou, X., Bai, C., Sun, X., Gong, X., Yang, Y., Chen, C., et al. (2017). Puerarin Attenuates Renal Fibrosis by Reducing Oxidative Stress Induced-Epithelial Cell Apoptosis via MAPK Signal Pathways In Vivo and In Vitro. Ren. Fail. 39, 423-431. doi:10.1080/0886022x.2017.1305409

Zhou, Y., Jiang, S. M., Li, L., Wang, Y., Ding, L., Liu, C. X., et al. (2020). Efficacy and Safety of Tanshinone for Chronic Kidney Disease: A Meta-Analysis. Evid. Based Complement. Alternat Med. 2020, 3091814. doi:10.1155/2020/ 3091814

Zhu, B., Wang, Y., Jardine, M., Jun, M., Lv, J. C., Cass, A., et al. (2013). Tripterygium Preparations for the Treatment of CKD: a Systematic Review and Meta-Analysis. Am. J. Kidney Dis. 62, 515-530. doi:10.1053/ j.ajkd.2013.02.374
Zhu, L., Han, J., Yuan, R., Xue, L., and Pang, W. (2018). Berberine Ameliorates Diabetic Nephropathy by Inhibiting TLR4/NF-Kb Pathway. Biol. Res. 51, 9. doi:10.1186/s40659-018-0157-8

Conflict of Interest: The authors declare that the research was conducted in the absence of any commercial or financial relationships that could be construed as a potential conflict of interest.

Publisher's Note: All claims expressed in this article are solely those of the authors and do not necessarily represent those of their affiliated organizations, or those of the publisher, the editors and the reviewers. Any product that may be evaluated in this article, or claim that may be made by its manufacturer, is not guaranteed or endorsed by the publisher.

Copyright (c) 2021 Shao, Ye, Bayliss and Zhuang. This is an open-access article distributed under the terms of the Creative Commons Attribution License (CC BY). The use, distribution or reproduction in other forums is permitted, provided the original author(s) and the copyright owner(s) are credited and that the original publication in this journal is cited, in accordance with accepted academic practice. No use, distribution or reproduction is permitted which does not comply with these terms. 